\title{
MM 45289, A POTENT GLYCOPEPTIDE ANTIBIOTIC WHICH INTERACTS WEAKLY WITH DIACETYL-L-LYSYL-D-ALANYL-D-ALANINE
}

\author{
Valerie M. Good, Michael N. Gwynn and David J. C. Knowles* \\ Beecham Pharmaceuticals Research Division, \\ Brockham Park, Betchworth, Surrey RH3 7AJ, UK
}

(Received for publication October 26, 1989)

MM 45289 (A82846A, eremomycin), a glycopeptide antibiotic of the vancomycin type, was confirmed to have improved antibacterial activity over vancomycin. However its affinity $(\mathrm{Ka})$ for the target site peptide mimetic diacetyl-L-lysyl-D-alanyl-D-alanine (DALAA) was 23-fold lower. Concentrations of DALAA required to reverse the antibacterial activity of MM 45289 were in the order of 10 to 50 -fold higher than for vancomycin. These results have implications for both mode of action studies and mechanism-based screening strategies for this class of antibiotic.

MM 45289 (A82846A, eremomycin) is a potent glycopeptide antibiotic closely related to vancomycin which has been isolated from strains of Amycolatopsis orientalis ${ }^{1 \sim 4)}$ (Fig. 1). Glycopeptide antibiotics are considered to act on bacterial cell wall biosynthesis at the level of transpeptidation/transglycosylation by binding to peptides containing a terminal D-alanyl-D-alanine residue, these being precursors to or the actual

Fig. 1. Structures of MM 45289 and vancomycin ${ }^{4,5)}$.

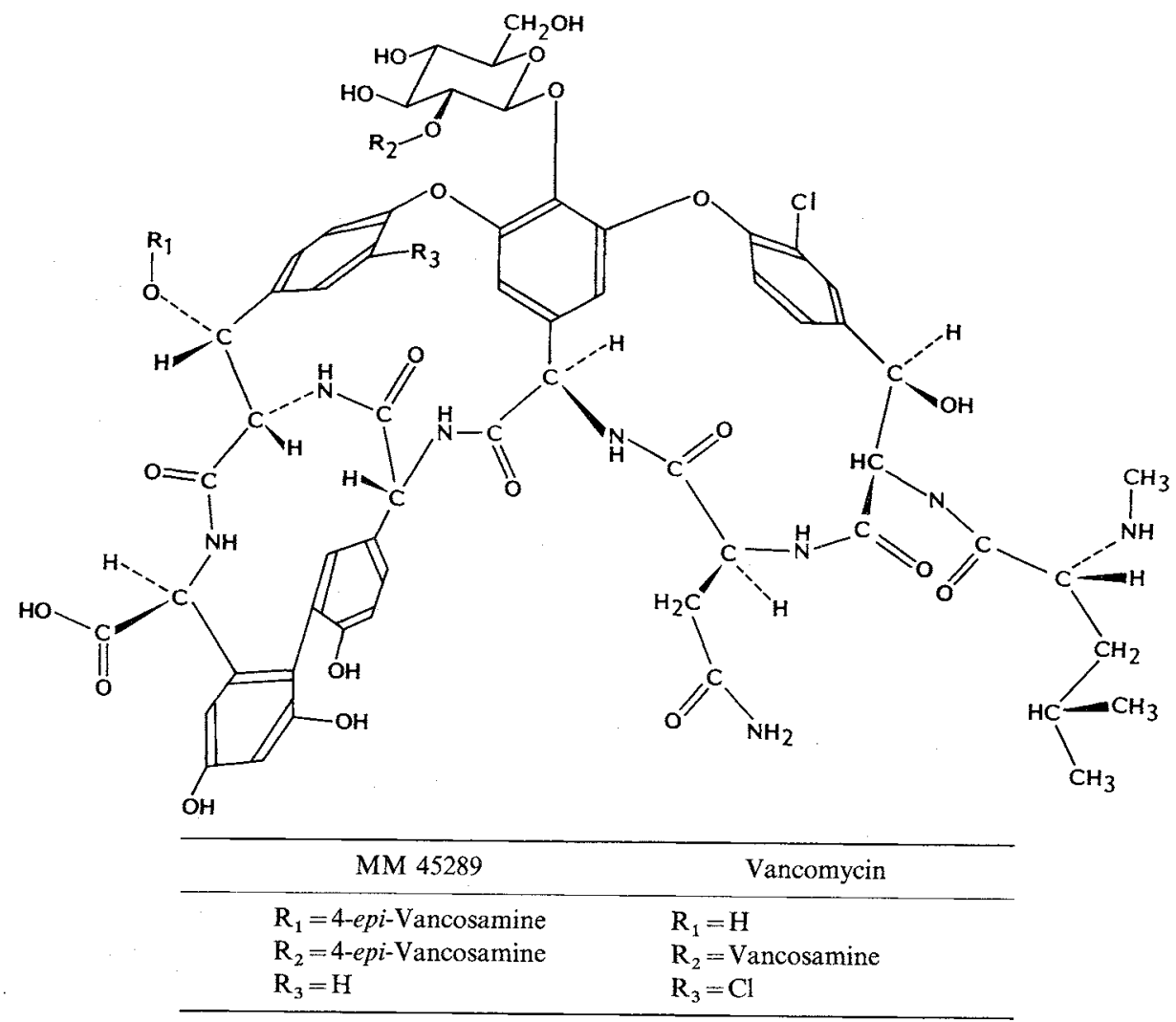


substrate for the transpeptidation reaction necessary for the formation of a rigid cell wall structure ${ }^{5)}$. Much work has been published on the nature of this binding and recently it was established that the affinity of close analogues of vancomycin for a model peptide ligand (diacetyl-L-lysyl-D-alanyl-D-alanine: DALAA) correlated with their antibacterial potency ${ }^{6}$. Indeed, the ability of glycopeptides to bind to DALAA prompted the design of a highly specific, mechanism-based screen using reversal or antagonism of antibacterial activity by DALAA to detect producers of this class of antibiotic from soil samples ${ }^{7)}$.

In this paper we report on the antibacterial activity of MM 45289 and its interaction with DALAA. The relevance of these results to the use of the DALAA reversal screen in the search for new glycopeptide antibiotics is also discussed.

\section{Materials and Methods}

\section{Antibiotics and Other Reagents}

DALAA, L-alanyl-D-glutaminyl-L-lysyl-D-alanyl-D-alanine (AGLAA), L-alanyl-D-isoglutaminyl-Llysyl-D-alanyl-D-alanine (AILAA) and vancomycin were obtained from Sigma. MM 45289 was isolated from A. orientalis NCIB 12531 at Brockham Park; its structure being identical to that reported for A82846A and eremomycin (M. GiLPIN; personal communication).

\section{Determination of Antibiotic MIC}

The activity of vancomycin and MM 45289 against 36 clinical isolates of coagulase-positive and negative Staphylococci and against Bacillus subtilis ATCC 6633 were assessed by conventional agar and broth microdilution MIC tests. The media used were nutrient, Isosensitest and Diagnostic Sensitivity Test (DST) agars and Isosensitest (IST) broth and Nutrient Broth No. 2 (Oxoid). Inocula were prepared from 18-hour broth cultures and contained between $10^{5}$ to $10^{6} \mathrm{cfu}$. MICs were read after 18 hours at $37^{\circ} \mathrm{C}$ incubation.

\section{Potentiation and Antagonism of Antibacterial Activity}

The effect of peptide ligands on the antibacterial activity of vancomycin and MM 45289 was investigated using agar zone diffusion assays and broth microdilution tests. Paper disc diffusion assays with $B$. subtilis ATCC 6633 were carried out exactly as described by RAKE et al ${ }^{77}$, using $100 \mu \mathrm{g}$ DALAA per disc. Diffusion assays with Staphylococcus aureus W8184, a clinical isolate, were carried out on DST agar; glycopeptides and DALAA being added to $7 \mathrm{~mm}$ wells cut in the agar $(80 \mu \mathrm{l}$ volume). Inhibition zones were measured after 18 hours at $37^{\circ} \mathrm{C}$ and glycopeptide potency calculated by reference to inhibition zone diameters of standard concentrations of glycopeptide. Conventional checkerboard broth microdilution tests employing serial 2-fold dilutions of glycopeptide and peptide ligand were carried out in IST broth. The components were stored in the microtitre trays for 18 hours at $4^{\circ} \mathrm{C}$ before bacteria at $10^{6} \mathrm{cfu} /$ well were inoculated. Glycopeptide MICs for each concentration of ligand were read after a further 18 hours at $37^{\circ} \mathrm{C}$.

\section{UV Difference Spectroscopy}

Antibiotic solutions $(2.5 \mathrm{ml}$ at $0.136 \mathrm{~mm}$ in $0.02 \mathrm{M}$ sodium citrate buffer, $\mathrm{pH} 5.2)$ were placed in the sample and reference cells of a Perkin Elmer 554 spectrophotometer. DALAA ( $1 \sim 20 \mu 1$ of $33.6 \mathrm{~mm}$ solution $)$ was added to the sample cell and buffer to the reference cell. The magnitude of the difference in absorbance between 282 and $293 \mathrm{~nm}$ was measured at $25 \pm 2^{\circ} \mathrm{C}$. Association constants were then deduced from Scatchard plots assuming a simple equilibrium: Glycopeptide + peptide $=$ glycopeptide-peptide complex ${ }^{8)}$. Antibiotic concentrations were determined by UV with a value of $\mathrm{E}_{1 \mathrm{~m}}^{1 \%}=45.0$ at $280 \mathrm{~nm}$ for both vancomycin and MM 45289.

\section{Results}

Antibacterial Activity of Vancomycin and MM 45289

MM 45289 was consistently more active than vancomycin against Staphylococci and B. subtilis in a 
Table 1. Antibacterial activity.

\begin{tabular}{clll}
\hline \multirow{2}{*}{ Organism } & Medium & \multicolumn{2}{c}{ MIC $(\mu \mathrm{g} / \mathrm{ml})$} \\
\cline { 3 - 4 } Staphylococcus spp. $n=36$ & & Vancomycin & MM 45289 \\
& NA & $3.7(2 \sim 8)^{\mathbf{a}}$ & $1.6(1 \sim 4)$ \\
& NB & $2.7(2 \sim 4)$ & $0.7(0.1 \sim 4)$ \\
Bacillus subtilis ATCC 6633 & ISTA & $2.4(1 \sim 8)$ & $(1.7) 4)$ \\
& ISTB & $1.4(1 \sim 2)$ & $0.3(0.1 \sim 2)$ \\
& DSTA & $2.6(2 \sim 4)$ & $(1.4) 1 \sim 4)$ \\
& NA & 1.0 & 0.25 \\
& NB & 0.5 & 0.03 \\
& ISTA & 0.5 & 0.25 \\
\hline
\end{tabular}

Comparative activity of vancomycin and MM 45289 against Staphylococci and B. subtilis ATCC 6633 by agar and broth microdilution (see Materials and Methods).

NA: nutrient agar, NB: nutrient broth, ISTA: Isosensitest agar, ISTB: Isosensitest broth, DSTA: Diagnostic Sensitivity Test agar.

a Staphylococcal data is presented as geometric mean MIC (range) for 36 strains.

range of different media (Table 1). Little difference in the susceptibility of the various staphylococcal strains was observed, as evidenced by the narrow range of MICs obtained. The difference between MM 45289 and vancomycin MICs tended to be greater in broth than on agar.

\section{Effect of DALAA on Glycopeptide Potency Estimated by Bioassay}

In preliminary agar diffusion assays using $S$. aureus W8184, $100 \mu \mathrm{g}$ DALAA completely abolished the inhibition zone surrounding a paper disc containing $12.5 \mu \mathrm{g}$ vancomycin. However, the same concentration of DALAA tended to increase the zone diameter around a similar disc of MM 45289 .

This phenomenon was studied further; results being expressed in terms of antibacterial potency relative to that of standards without DALAA (Fig. 2). As expected, the amount of DALAA required to antagonise vancomycin increased with the concentration of glycopeptide. At none of the concentrations tested did DALAA potentiate the activity of vancomycin. In marked contrast, DALAA did potentiate the activity of MM 45289 by between 20 and $90 \%$. At higher concentrations, DALAA antagonised the activity of MM 45289. The concentration at which it did so was between 10 and 20 times that required for similar concentrations of vancomycin.

Similar results were seen using the previously published mechanism-based screen employing $B$. subtilis. DALAA $(100 \mu \mathrm{g})$ reduced the zone of inhibition around vancomycin discs but enhanced that around discs containing MM 45289 (Fig. 3). Furthermore, paper discs dipped in the culture medium from $A$. orientalis. NCIB 12531 also gave an increased zone diameter in the presence of DALAA (Fig. 3).

\section{Effect of DALAA and Two Pentapeptides on Glycopeptide MICs}

Reversal experiments were also carried out in conventional microdilution susceptibility tests with $S$. aureus W8184 and B. subtilis ATCC 6633. Again, the activity of MM 45289 was less affected by DALAA than that of vancomycin. The lowest concentration of DALAA which resulted in an increase in the MIC of MM 45289 was approximately ten to fifty times that affecting vancomycin MICs (Fig. 4). No evidence of potentiation of MM 45289 by DALAA was seen in terms of a decrease in MIC. Preliminary microdilution MIC tests with two other peptide ligands (AILAA and AGLAA) gave results similar to those with DALAA (Table 2). For example, at $512 \mu \mathrm{M}(190 \sim 250 \mu \mathrm{g})$ all three ligands raised the vancomycin MIC for S. aureus 
Table 2. Reversal of antibacterial activity by peptide ligands.

\begin{tabular}{|c|c|c|c|c|c|c|c|c|c|}
\hline \multirow[b]{2}{*}{ Ligand } & \multirow[b]{2}{*}{ Glycopeptide } & \multicolumn{8}{|c|}{ Glycopeptide MIC $(\mu \mathrm{M})$} \\
\hline & & \multicolumn{3}{|c|}{ (conc of ligand $(\mu \mathrm{M})$ ) } & $\begin{array}{r}32 \\
\text { (cone } 0\end{array}$ & $\begin{array}{c}64 \\
d(\mu\end{array}$ & 128 & 256 & 512 \\
\hline DALAA & Vancomycin & 2 & 4 & 4 & 8 & 16 & 32 & 64 & $>64$ \\
\hline DALAA & MM 45289 & 1 & 1 & 1 & 2 & 2 & 2 & 8 & 8 \\
\hline AILAA & Vancomycin & 2 & 4 & 4 & 8 & 8 & 16 & 32 & 64 \\
\hline AILAA & MM 45289 & 1 & 1 & 2 & 1 & 1 & 4 & 2 & 8 \\
\hline AGLAA & Vancomycin & 2 & 4 & 4 & 8 & 16 & 16 & 64 & $>64$ \\
\hline AGLAA & MM 45289 & 1 & 1 & 1 & 1 & 1 & 2 & 2 & 4 \\
\hline
\end{tabular}

Effect of peptide ligands on glycopeptide MICs to Staphylococcus aureus W8184 by broth microdilution (1 $\mu \mathrm{M}$ glycopeptide: $c 1.5 \mu \mathrm{g} / \mathrm{ml}$ ).

Fig. 2. Effect of DALAA on the potency of vancomycin and MM 45289 by hole-in-plate bioassay using Staphylococcus aureus W8184.

Open symbols: Vancomycin, closed symbols: MM 45289. $\bigcirc$ and $\bullet: 5 \mu \mathrm{g} / \mathrm{well}, \Delta$ and $\Delta: 20 \mu \mathrm{g} / \mathrm{well}, \square$ and ⿷: $80 \mu \mathrm{g} /$ well, $\nabla$ and $\nabla: 320 \mu \mathrm{g} /$ well.

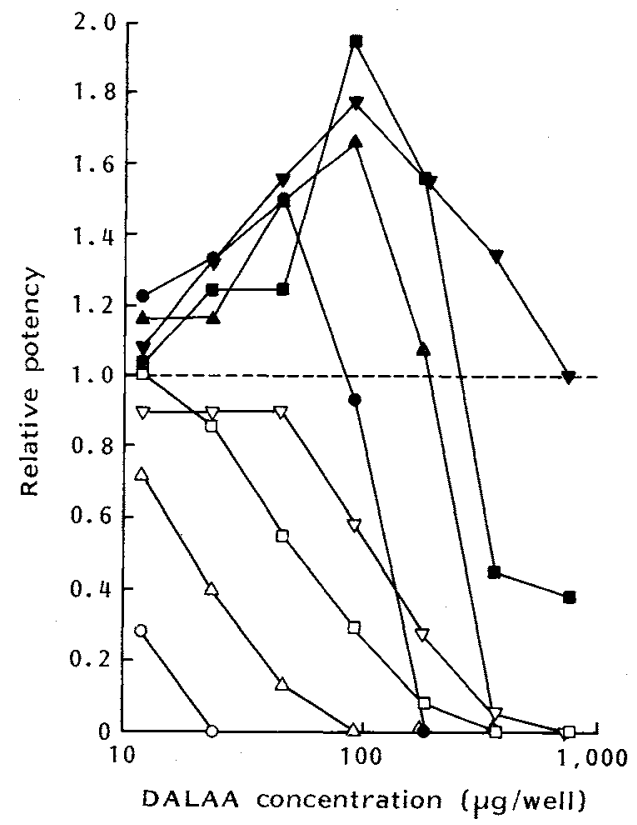

Glycopeptides were tested at 5, 20, 80 and $320 \mu \mathrm{g} /$ well and the relative potency in different concentrations of DALAA calculated by reference to a standard line in the absence of DALAA.
Fig. 3. Zone diameters of glycopeptide standard lines using the Bacillus subtilis ATCC 6633 mechanismbased glycopeptide screening protocol ${ }^{7)}$.

Open symbols: No DALAA, closed symbols: $100 \mu \mathrm{g}$ DALAA per disc, $O$ and $\bullet$ : vancomycin, $\triangle$ and $\Delta$ : MM 45289, $\square$ and $\mathbf{a}$ : culture medium of $\mathrm{MM}$ 45289-producer Amycolatopsis orientalis NCIB 12531.

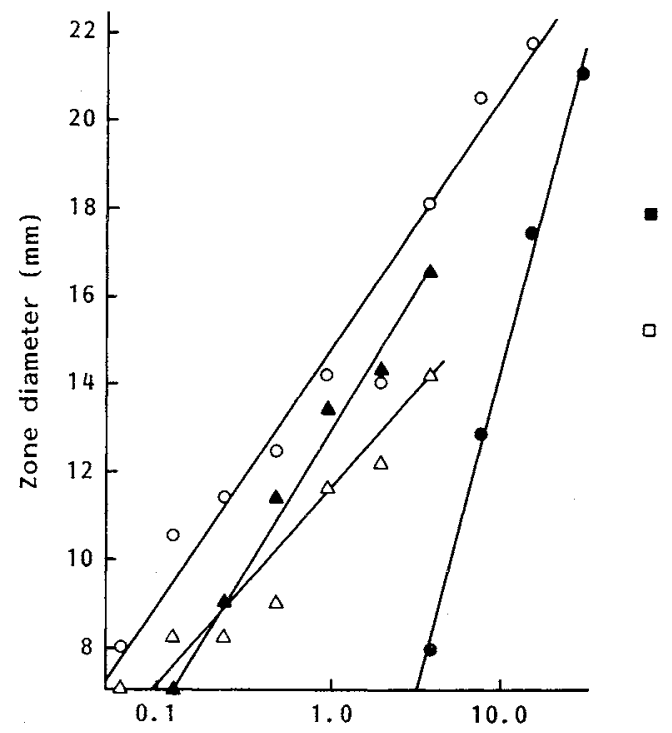

Glycopeptide concentration ( $\mu \mathrm{g} / \mathrm{disc}$ )

W8184 from 2 to $>64 \mu \mathrm{M}$ (>32-fold) whereas the $\mathrm{MIC}$ of $\mathrm{MM} 45289$ was raised from 1 to $4 \mu \mathrm{M}$ (AGLAA) or $8 \mu \mathrm{M}$ (AILAA and DALAA).

\section{UV Difference Spectroscopy}

Representative Scatchard plots are shown in Fig. 5 from which DALAA affinity constants for MM 45289 and vancomycin were obtained. The mean $K a$ value from repeated experiments for vancomycin was $0.913 \times 10^{6} \mathrm{M}^{-1}$ (standard deviation $=0.213 \times 10^{6} ; n=3$ ) and that for MM 45289 was $0.039 \times 10^{6} \mathrm{M}^{-1}$ 
Fig. 4. Effect of DALAA on glycopeptide MICs against Staphylococcus aureus W8184 and Bacillus subtilis ATCC 6633 .

(A) S. aureus, (B) B. subtilis. O vancomycin, $\triangle \mathrm{MM} 45289$.

(A)

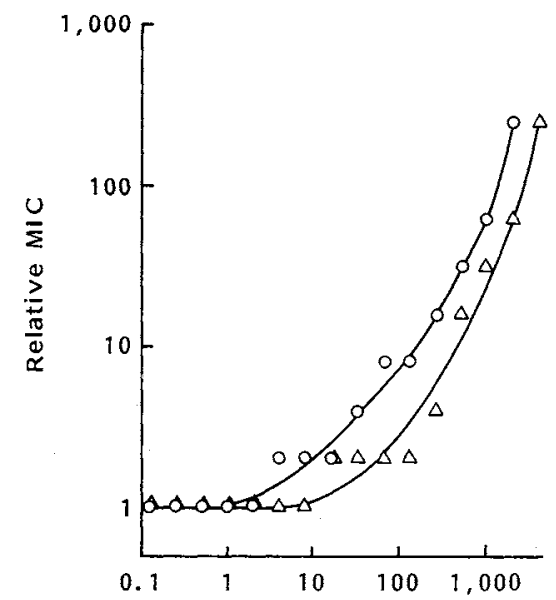

(B)

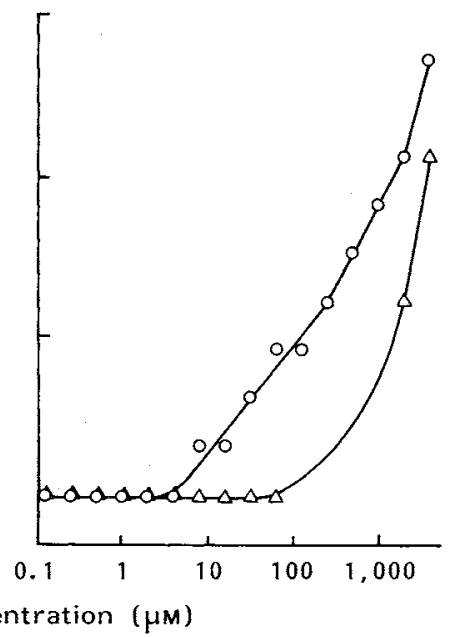

MICs are expressed relative to those without DALAA. Microdilution test in Isosensitest Broth.

Fig. 5. Representative Scatchard plots of vancomycin and MM 45289 binding to DALAA.

O Vancomycin, $\triangle \mathrm{MM} 45289$.

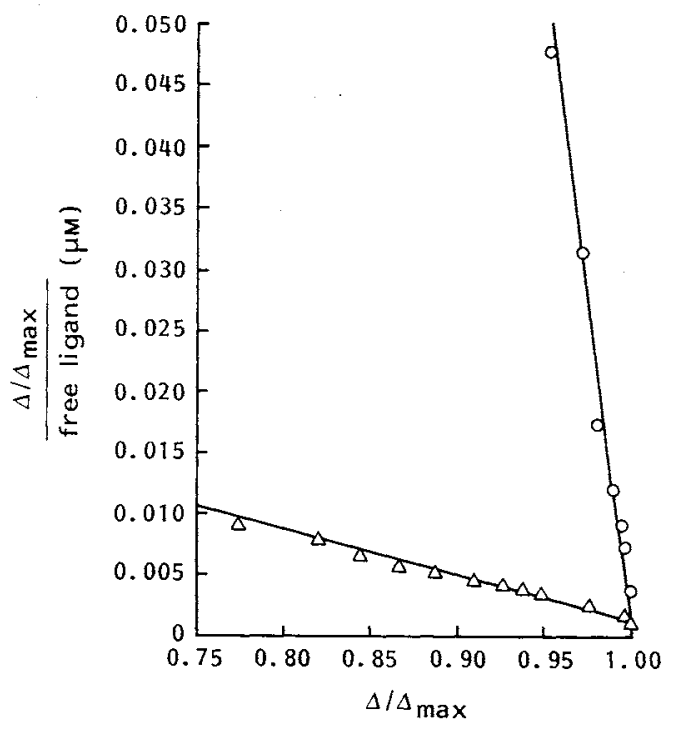

Data based on absorbance change from 293 to $282 \mathrm{~nm}$

in the presence of ligand (see Materials and Methods).

(standard deviation $=0.009 \times 10^{6} ; n=6$ ); a 23-fold difference in affinity.

\section{Discussion}

Vancomycin and MM 45289 share the same heptapeptide nucleus but differ in the number of chlorine atoms, the stereochemistry of the basic sugar moiety in the central disaccharide unit (epi-vancosamine in MM 45289) and, in MM 45289, the presence of a second epi-vancosamine moiety attached directly to the heptapeptide. These differences result in MM 45289 being more active than vancomycin in vitro; a finding consistent with other studies ${ }^{4,9)}$. However, its interaction with the tripeptide DALAA, the two pentapeptides AILAA and AGLAA, and its affinity for DALAA, are much reduced.

There was good quantitative agreement between the antibacterial reversal data and relative affinity constant calculated by UV absorbance changes. The reversal experiments indicated that the amount of DALAA required to reverse the activity of MM 45289 was $10 \sim 20$ (zone diffusion) and $10 \sim 50$ (microdilution $\mathrm{MIC}$ ) times that required for vancomycin. The difference in calculated $K a$ 's was 23-fold.

The lack of correlation between MIC and DALAA affinity for vancomycin and MM 45289 implies that this tripeptide is limited in its use as a simple model of the glycopeptide peptidoglycan target. The poor reversal of MM 45289 activity by 
AILAA and AGLAA suggest that the same may be true for these pentapeptides. The possibility that MM 45289 has a second mode of action is unlikely since it is active against neither L-form Staphylococci nor Enterococci expressing the $39 \mathrm{Kd}$ vancomycin resistance determinant specific for glycopeptide antibiotics ${ }^{10}$ ) (unpublished observations).

The potentiation of MM 45289 activity by low concentrations of DALAA, revealed in agar diffusion tests, was unexpected. The level of potentiation was slight; less than 2-fold and therefore not expected to show up in conventional MIC tests. The mechanism(s) underlying this potentiation are not known but possible explanations include cooperative interaction with cell wall precursors in the presence of exogenous ligand. Alternatively, since DALAA is a substrate for purified staphylococcal transpeptidase ${ }^{11)}$, it may be able to undergo a similar reaction in vivo. This would result in a reduction in peptidoglycan cross-linking and may synergise with glycopeptide activity. Such synergy with DALAA would be seen with glycopeptides such as MM 45289, which are highly potent and yet have a low affinity for the ligand. However, glycopeptides with a higher affinity for DALAA such as vancomycin would not be expected to show such synergy; both their own intrinsic activity and the ligand's involvement in transpeptidation reactions being mutually inhibited.

Whatever the reason for the observed potentiation between MM 45289 and DALAA on bioassay, the failure of the mechanism-based DALAA reversal screen to detect MM 45289 in the culture medium of the producer culture (Fig. 3) is significant. Modification of such an approach would appear to be indicated in order to ensure the detection of glycopeptides, such as MM 45289, with potent antibacterial activity coupled with a poor affinity for DALAA.

\section{References}

1) Athalye, M.; A. Elson, M. Gilpin \& L. Jefrries (Beecham Group ple): Antibiotic compounds. Eur. Pat. Appl. 0309161, Nov. 28, 1988

2) Athalye, M.; S. Holland, D. Lewis, I. Pallet \& D. J. Winstanley: MM 45289 and MM 47756, two glycopeptide antibiotics produced by a new strain of Amycolatopsis ("Nocardia") orientalis. Taxonomy and fermentation studies. Int. J. Syst. Bacteriol., in preparation

3) Nagarajan, R.; D. M. Berry, A. H. Hunt, J. L. Occolowitz \& A. A. Schabel: Conversion of antibiotic A82846B to orientacin A and structural relationships of related antibiotics. J. Org. Chem. 54: 983 986, 1989

4) Brazhnikova, M. G.; N. N. Lomakina \& T. F. Beronikova: Eremomycin: A new glycopeptide antibiotic. In Progress in Industrial Microbiology 27. Bioactive Metabolites from Microorganisms. Eds., M. E. Bushell \& U. GrÄFE, pp. 163 175, Elsevier Science Publishers B. V., 1989

5) BARNA, J. C. J. \& D. H. Williams: The structure and mode of action of glycopeptide antibiotics of the vancomycin group. Ann. Rev. Microbiol. 38: 339 357, 1984

6) Kannan, R.; C. M. Harris, T. M. Harris, J. P. Waltho, N. J. Skelton \& D. H. Williams: Function of the aminosugar and $N$-terminal amino acid of the antibiotic vancomycin in its complexation with cell wall peptides. J. Am. Chem. Soc. 110: 2946 2953, 1988

7) Rake, J. B.; R. Gerber, R. J. Mehta, D. J. Newman, Y. K. Oh, C. Phelen, M. C. Shearer, R. D. Sitrin \& L. J. NisBEt: Glycopeptide antibiotics: A mechanism-based screen employing a bacterial cell wall receptor mimetic. J. Antibiotics 39: 58 67, 1986

8) Nieto, M. \& H. R. Perkins: Physicochemical properties of vancomycin and iodovancomycin and their complexes with diacetyl-L-lysyl-D-alanyl-D-alanine. Biochem. J. 123: 773 787, 1971

9) Counter, F. T.; P. W. ENSMinger \& C. Y. E. Wu: A82846, a new glycopeptide complex, produced by Amycolatopsis orientalis. 4. Microbiological evaluation. Program and Abstracts of the 28th Intersci. Conf. on Antimicrob. Agents Chemother., No. 977, p. 283, Los Angeles, Oct. 23 26, 1988

10) Uttley, A. H. C.; C. H. Collins, J. Naidoo \& R. C. George: Vancomycin-resistant enterococci. Lancet 1988-I: $57 \sim 58,1988$

11) Kozarich, J. W. \& J. L. Strominger: A membrane enzyme from Staphylococcus aureus which catalyses transpeptidase, carboxypeptidase, and penicillinase activities. J. Biol. Chem. 253: 1272 1278, 1978 